\title{
Friedreich Ataxia Mouse Models with Progressive Cerebellar and Sensory Ataxia Reveal Autophagic Neurodegeneration in Dorsal Root Ganglia
}

\author{
Delphine Simon, ${ }^{1}$ Hervé Seznec, ${ }^{1}$ Anne Gansmuller, ${ }^{1}$ Nadège Carelle, ${ }^{1}$ Philipp Weber, ${ }^{1}$ Daniel Metzger, ${ }^{1}$ Pierre Rustin, ${ }^{2}$ \\ Michel Koenig, ${ }^{1}$ and Hélène Puccio ${ }^{1}$ \\ ${ }^{1}$ Institut de Génétique et de Biologie Moléculaire et Cellulaire, Centre National de la Recherche Scientifique/Institut National de la Santé et de la Recherche \\ Médicale (INSERM)/Université Louis Pasteur, 67404 Illkirch cedex, CU de Strasbourg, France, and ²Unité de Recherches sur les Handicaps Génétiques de \\ l'Enfant, INSERM U393, Hôpital Necker-Enfants Malades, 75015 Paris, France
}

Friedreich ataxia (FRDA), the most common recessive ataxia, is characterized by degeneration of the large sensory neurons of the spinal cord and cardiomyopathy. It is caused by severely reduced levels of frataxin, a mitochondrial protein involved in iron-sulfur cluster (ISC) biosynthesis. Through a spatiotemporally controlled conditional gene-targeting approach, we have generated two mouse models for FRDA that specifically develop progressive mixed cerebellar and sensory ataxia, the most prominent neurological features of FRDA. Histological studies showed both spinal cord and dorsal root ganglia (DRG) anomalies with absence of motor neuropathy, a hallmark of the human disease. In addition, one line revealed a cerebellar granule cell loss, whereas both lines had Purkinje cell arborization defects. These lines represent the first FRDA models with a slowly progressive neurological degeneration. We identified an autophagic process as the causative pathological mechanism in the DRG, leading to removal of mitochondrial debris and apparition of lipofuscin deposits. These mice therefore represent excellent models for FRDA to unravel the pathological cascade and to test compounds that interfere with the degenerative process.

Key words: behavior; degeneration; dorsal root ganglion; DRG; mitochondria; neuropathology; Purkinje cell; mouse model; Friedreich ataxia

\section{Introduction}

Friedreich ataxia (FRDA), the most frequent hereditary ataxia, is an autosomal recessive neurodegenerative disease characterized by progressive ataxia and dysarthria, sensory neuropathy, deep sensory impairment, and signs of pyramidal tract involvement (Harding, 1981). Extraneurological symptoms include cardiomyopathy (Harding and Hewer, 1983) and diabetes mellitus (Finocchiaro et al., 1988). Pathologic changes occur first in dorsal root ganglia (DRG), with loss of large sensory neurons, followed by, in spinal cord, axonal degeneration in posterior columns, pyramidal, and spinocerebellar tracts as well as atrophy and neuronal loss in Clarke's column (Hughes et al., 1968; Oppenheimer and Esiri, 1992). Mild degenerative changes are observed in medulla, cerebellum, and pons (Koeppen, 1998).

FRDA is caused by partial loss of function of frataxin, a ubiq-

Received 0ct. 7, 2003; revised Nov. 28, 2003; accepted Jan. 1, 2004.

This work was supported by funds from the European Community (Contract QLG1-CT-1999-0584), the Muscular Dystrophy Association (Contract 3184), the Association Française contre l'Ataxie de Friedreich (H.S.), Institut National de la Santé et de la Recherche Medicale, Centre National de la Recherche Scientifique, and the Hôpital Universitaire de Strasbourg. We thank J. L. Mandel and members of his laboratory for discussions and comments; L. Reutenauer, N. Lagarde, S. Bronner, and J. Hergueux for technical support; J. L. Vonesch for conception of the software for footprint analysis; and $\mathrm{G}$. Yvert for advice on the rotarod test.

Correspondence should be addressed to Hélène Puccio, Institut de Génétique et de Biologie Moléculaire et Cellulaire, 1 rue Laurent Fries BP 10142, 67404 Illkirch cedex, France. E-mail: hpuccio@igbmc.u-strasbg.fr.

DOI:10.1523/JNEUROSCI.4549-03.2004

Copyright $\odot 2004$ Society for Neuroscience $\quad$ 0270-6474/04/241987-09\$15.00/0 uitously expressed mitochondrial protein, through inhibition of transcriptional elongation by a GAA triplet expansion within the first intron of the gene (Campuzano et al., 1996). In the nervous system, frataxin is highly expressed in spinal cord, particularly in DRG and at lower levels in cerebellum (Koutnikova et al., 1997). The function of frataxin has recently been linked to iron-sulfur cluster (ISC) biosynthesis in yeast (Huynen et al., 2001; Lutz et al., 2001; Duby et al., 2002; Muhlenhoff et al., 2002). Its absence causes generalized ISC-containing enzyme deficiency, which is also observed in frataxin-deficient patients and mice, and eventually leads to iron homeostasis disturbances and oxidative stress (Puccio and Koenig, 2002). In FRDA patients, iron deposits have been observed in heart (Lamarche et al., 1993). Moreover, deficiencies of ISC-containing respiratory chain complexes I-III and aconitases have been demonstrated in heart biopsies (Rotig et al., 1997) and in the DRG (aconitases) (Bradley et al., 2000). Finally, there is evidence of increased oxidative stress in patients with elevated urine 8-hydroxy-2' -deoxyguanosine and serum malondialdehyde levels (indicative of DNA damage and lipid peroxidation, respectively) (Emond et al., 2000; Schulz et al., 2000) and an impaired response to oxidative challenge in patients' fibroblasts (Chantrel-Groussard et al., 2001).

We have recently generated two conditional knock-out models for FRDA, a cardiac and a neuronal-cardiac model, which reproduce important pathophysiological features of the human 
disease (Puccio et al., 2001). Although the neuronal-cardiac mice show a specific progressive ataxia with loss of proprioception, the severity of the disease prevented us from evaluating the primary sites of neurodegeneration and performing therapeutic trials. Indeed, the mice have a short life expectancy of $25 \mathrm{~d}$ with lesions not seen in the human disease (liver and spongiform cortical lesions), preventing cell-specific degeneration mechanisms studies.

To obtain specific and progressive neurological models for FRDA, we generated inducible knock-out mouse models using two transgenic lines (having distinct neuronal specificities) expressing the tamoxifen-dependent recombinase $\left(\right.$ Cre-ER $\left.{ }^{\mathrm{T}}\right)$ under the mouse Prion protein (Prp) promoter, thus enabling us to spatiotemporally control somatic mutagenesis of conditional alleles of the target genes (Weber et al., 2001). We show here that ablation of frataxin in adult mice leads to progressive neurological symptoms resembling FRDA. This study reports the detailed characterization of both inducible neurological models and a newly identified pathological mechanism found in DRG, one of the primary affected sites in FRDA.

\section{Materials and Methods}

Animals. 28.4 and 28.6 Prp-Cre-ER ${ }^{\mathrm{T}}$ (Weber et al., 2001), Frda ${ }^{+/ \mathrm{L}-}$, and Frda ${ }^{\mathrm{L} 2 / \mathrm{L} 2}$ mice (L2 allele is derived from original L3 allele by Cremediated excision of the Neomycine cassette) and mouse genotyping were as described (Puccio et al., 2001). The Prp-Cre-ER ${ }^{\mathrm{T}}$ transgene, under the control of the prion protein promoter, expresses the Cre recombinase fused with the ligand binding domain of a mutant estrogen receptor with a nuclear translocation that is activated only through tamoxifen binding. Animals were treated with $1 \mathrm{mg}$ of tamoxifen (Sigma, Deisenhofen, Germany) by intraperitoneal injection for 5 consecutive days (Metzger and Chambon, 2001) at 4 weeks of age, 1 week after weaning. All methods used in this work are in accordance with the Guide for the Care and Use of Laboratory Animals published by the National Institutes of Health (NIH Publication No. 85-23, revised 1996).

Western blot analysis. Protein extractions, Western blot, and estimation of immunoblot residual level of frataxin using a polyclonal antifrataxin antibody (R1250 purified sera, 1:1000) (Puccio et al., 2001) and a monoclonal anti- $\beta$-tubulin (1:5000; Boehringer Mannheim, Mannheim, Germany) were as described previously (Puccio et al., 2001). Homozygous $\left(F r d a^{\mathrm{L} 2 / \mathrm{L} 2}\right)$ and heterozygous $\left(\mathrm{Frda}^{+/ \mathrm{L}-}\right)$ mice negative for Prp-Cre-ER ${ }^{\mathrm{T}}$ expression were used as controls. Measurements were performed on heart, liver, pancreas, muscle, spleen, kidney, lung, cerebellum, brainstem, brain, and spinal cord at least three times on six animals from different litters.

Footprint analysis. After coating of hindfeet with nontoxic ink, mice were allowed to walk through a tunnel ( $50 \mathrm{~cm}$ long, $9 \mathrm{~cm}$ wide, $6 \mathrm{~cm}$ high) with paper lining the floor (Clark et al., 1997). The footprint pattern generated was scored for five parameters. Three consecutive steps define a triangle (see Fig. 2f) with the segments scored from [1] to [6]. Step length, the average distance of forward movement between alternate steps, is defined as the distance of travel through the tunnel divided by the number of steps. Sigma, describing the regularity of step length, is defined as the standard variation of all right-right and left-left step distance (as [3]). Gait width, the average lateral distance between opposite left and right steps, is determined by measuring the perpendicular distance of a given step to a line connecting its opposite preceding and succeeding steps (as [6]). Alternation coefficient, describing the uniformity of step alternation, is calculated by the mean of the absolute value of 0.5 minus the ratio of right-left distance to right-right step distance for every leftright step pair [as $0.5-([4] /[3])]$. Linearity, average change in angle between consecutive right-right steps is calculated by drawing a line perpendicular to direction of travel ([1]), starting at first right footprint. After determining angle between this perpendicular line and each subsequent right footprint, differences in angle were estimated between each consecutive step pair, and the average of absolute values of all angles was calculated (angle *). A high linearity score is indicative of nonlinear movement.

Accelerating rotating rod test. Coordination, equilibrium, and motor skill acquisition were tested using an accelerated rotating rod (Panlab, Barcelona, Spain) test as described previously (Clark et al., 1997). Briefly, mice at 10,20,30, and 40 weeks were placed on the rod in four trials every day for a period of $4 \mathrm{~d}$ (eight controls and eight mutants at each age). The rod accelerated from 4 to $40 \mathrm{rpm}$ in $5 \mathrm{~min}$ and remained at maximum speed for the next $5 \mathrm{~min}$. Animals were scored for their latency to fall (in seconds) for each trial and rested a minimum of 10 min between trials to avoid fatigue and exhaustion. Results were analyzed by a repeatedmeasure ANOVA test considering three factors: days (fixed); genotype (fixed); animals (variable), nested in genotype and crossed with days.

Muscular strength measurement. Mice placed on the grid of a dynamometer (BioSeb, Chaville, France) were pulled by their tails in the opposite direction. The maximal strength exerted by the mouse before losing grip was recorded. The mean of three measurements allowing 30 sec of recovery time between each of them was calculated.

Histology and immunohistochemistry. For histology, animals were intracardially perfused with Bouin or $4 \%$ paraformaldehyde (PFA) in $0.1 \mathrm{M}$ phosphate buffer (PBS), $\mathrm{pH} 7.3$, and the various tissues were dissected, fixed for several days, and embedded in paraffin. For brain and spinal cord analysis, the head and the column were decalcified in Jenkins solution (Gabe, 1968) during a period of 3 or $6 \mathrm{~d}$. The sequential slides $(7 \mu \mathrm{m})$ of the paraffin blocks were stained with hematoxylin and eosin (H\&E), Perls $\mathrm{Fe}^{3+}$ to detect iron (Puccio et al., 2001), or calbindin (1:1000; rabbit anti-calbindin D-28K; Swant, Bellinzona, Switzerland) to label the cytoplasmic region of Purkinje cells. For calbindin staining, sections were visualized using the Vectastain ABC Kit (Vector Laboratories, Burlingame, CA) as described in the manufacturer's protocol. For immunohistochemistry, the animals were intracardially perfused with $4 \%$ PFA in PBS. After overnight fixation, the spinal cord with DRG was dissected from the column, cryoprotected $24 \mathrm{hr}$ in $30 \%$ sucrose, and included in frozen specimen embedding medium (Cryomatrix; ThermoShandon, Pittsburgh, PA). Sections ( $7 \mu \mathrm{m})$ were mounted on gelatin-coated slides. Frataxin was detected by immunohistochemistry using rabbit polyclonal purified R1250 antibody (1:1000) followed by an anti-rabbit IgG Cy3conjugated secondary antibody (1:1000; Jackson Laboratory, Bar Harbor, ME).

Apoptosis in head and column sections from animals perfused with 4\% PFA in PBS was assessed by the in situ DNA end-labeling [terminal deoxynucleotidyl transferase-mediated biotinylated UTP nick end labeling (TUNEL)] method, following the manufacturer's directions (Apoptag, Intergen Co., Purchase, NY).

Electron microscopy. Animals were intracardially perfused with 2.5\% glutaraldehyde in PBS. After overnight fixation, the spinal cord with DRG was dissected from the column and fixed for 1 additional day. Tissues were rinsed in PBS, postfixed in 1\% osmium tetroxidePBS for $2 \mathrm{hr}$ at $4^{\circ} \mathrm{C}$, dehydrated, and embedded in Epon. Regions of interest were localized on $2 \mu \mathrm{m}$ sections stained with toluidine blue. Ultrathin sections from selected areas were stained with uranyl acetate and lead citrate and examined with a Philips 208 electron microscope, operating at $80 \mathrm{kV}$.

Electrophysiological recordings. Electromyographic recordings were performed by Neurofit (Strasbourg, France) using a Neuromatic 2000M apparatus (Dantec, Les Ulis, France). Mice were anesthetized with intraperitoneal injection of $60 \mathrm{mg} / \mathrm{kg}$ ketamine chlorhydrate (Imalgène 500; Rhône Biomerieux, Lyon, France). Distal latency and amplitude of M and $\mathrm{H}$ waves were recorded in the plantar hindpaw muscle after sciatic nerve stimulation. Sensory nerve conduction velocity of the caudal nerve was also recorded.

Respiratory chain investigation. Brain, brain stem, spinal cord, cerebellum, and heart of mice were immediately frozen dry in liquid nitrogen. The activities of respiratory chain enzyme complexes, cytochrome $c$ oxidase-complex IV (COX) and succinate dehydrogenase-complex II (SDH), were determined as described (Rustin et al., 1994). 
a

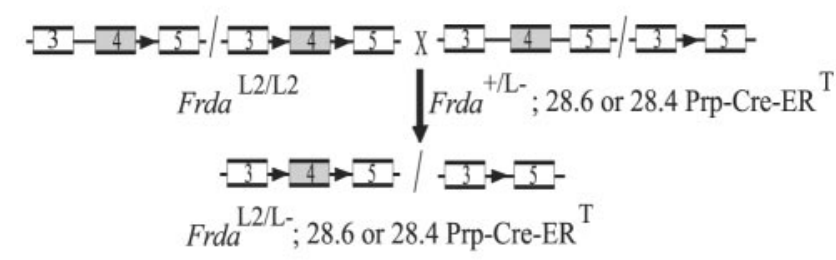

b
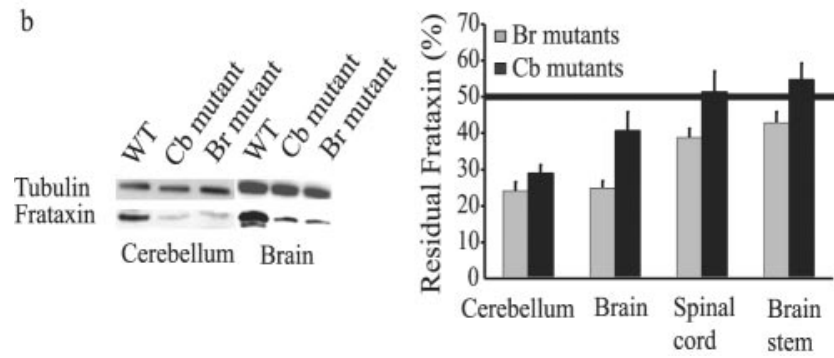

Figure 1. Br and Cb mutants show neuronal-specific frataxin-induced deletion. $a$, Schematic representation of the crossing to generate $\mathrm{Cb}$ and Br mutants (Frda ${ }^{\mathrm{L} / \mathrm{L}-} ; 28.4$ or 28.6 Prp-Cre$E R^{\top}$, respectively). Frda ${ }^{\mathrm{L} 2}$ allele has frataxin exon 4 flanked by loxP sequences (black arrow) (Puccio et al., 2001), and Frda ${ }^{\mathrm{L}-}$ allele is the (re-mediated exon 4 deleted allele. Boxes represent exons. $b$, Western blot analysis of cerebellum, brain (forebrain and midbrain), spinal cord, and brain stem extracts, probed with a polyclonal anti-frataxin antibody (R1250) and a tubulin antibody. Figures on the graph represent the mean of three immunoblots performed for six different mice from each line. The horizontal line corresponds to the $50 \%$ of expression level of frataxin in an Frda ${ }^{\mathrm{L} 2 / \mathrm{L}-}$ mouse with no Prp-Cre ER ${ }^{\top}$ expression. Representative blots performed on brain and cerebellum extracts of a control mouse presenting $100 \%$ of frataxin expression and of $\mathrm{Cb}$ and $\mathrm{Br}$ mutants are shown. Percentage of residual frataxin $=100 \times$ (mutant frataxin/ control frataxin)/(mutant tubulin/control tubulin).

\section{Results}

\section{Generation of mutant mice}

To induce neuron-specific deletion of frataxin, we bred mice homozygous for the conditional frataxin allele $\left(\mathrm{Frda}^{\mathrm{L} 2 / \mathrm{L} 2}\right)$ with mice heterozygous for the deletion of frataxin exon $4\left(\mathrm{Frda}^{+/ \mathrm{L}-}\right)$ carrying either the 28.4 Prp-Cre-ER ${ }^{\mathrm{T}(\mathrm{tg} / 0)}$ or the 28.6 Prp-Cre$\mathrm{ER}^{\mathrm{T}(\mathrm{tg} / 0)}$ transgene that expresses the tamoxifen-inducible recombinase under the Prp (Fig. 1a). Both Prp-Cre-ER ${ }^{\mathrm{T}}$ lines express Cre-ER ${ }^{\mathrm{T}}$ in the nervous system, but whereas the 28.4 line has a wide expression pattern, Cre-ER ${ }^{\mathrm{T}}$ expression of the 28.6 line is mostly restricted to the hippocampus and the cerebellum (Weber et al., 2001). Offspring were injected intraperitoneally with $1 \mathrm{mg}$ of tamoxifen for 5 consecutive days at 4 weeks of age, and tamoxifen-treated mice $\mathrm{Frda}^{\mathrm{L} 2 / \mathrm{L}-} ; 28.4$ or 28.6 Prp-Cre $\mathrm{ER}^{\mathrm{T}(\mathrm{tg} / 0)}$ correspond to animals subsequently denoted brain $(\mathrm{Br})$ or cerebellar (Cb) mutants, respectively. Southern blot analysis of DNA extracts from different tissues revealed deletion of the frataxin gene in the brain (corresponding to the forebrain and midbrain) and cerebellum with no deletion in non-neuronal tissues (heart, spleen, liver, lung, muscle, pancreas, kidney) (data not shown); however, dilution of neuronal cells in a mass of other cells such as glia hampers the quantification of neuron-specific deletion by this method. Direct quantification of frataxin by Western blot is a more reliable method, because frataxin is prominently expressed in neurons compared with other cells of the nervous system (Koutnikova et al., 1997). We found significant frataxin reduction in the cerebellum of both the $\mathrm{Br}$ and $\mathrm{Cb} \mathrm{mu}-$ tants, as well as in the brain, brain stem, and spinal cord of the $\mathrm{Br}$ mutants (Fig. 1b), and no significant reduction in non-neuronal tissues (data not shown), in agreement with the reported neuronal-specific pattern of expression of the Cre-ER ${ }^{\mathrm{T}}$ transgenes (Weber et al., 2001).

\section{Mutant animals develop loss of coordination without muscle weakness}

Although $\mathrm{Cb}$ and $\mathrm{Br}$ mutant animals exhibit normal growth and life expectancy, they develop an abnormal gait and a progressive loss of proprioception. Onset of clinical symptoms for both mutant lines was 25 weeks after tamoxifen injection (all time points mentioned hereafter are considered after tamoxifen injection unless noted otherwise), including mild coordination and balance deficits, with the hindlimbs being primarily affected. In addition, mutant animals showed delay in initiation of movement, reduced voluntary movements (hypokinesia), abnormal walking posture, and partial loss of proprioception. These abnormalities worsen progressively until the mutant animals display clear ataxia (between 40 and 50 weeks). Although both lines exhibit a rather similar clinical phenotype, the progression of the symptoms is more severe for the $\mathrm{Cb}$ mutants, with the appearance of tremors and reduction of spontaneous ambulation at 40 weeks and a complete lack of coordination and balance by 1 year of age. In contrast, $\mathrm{Br}$ mutants even at 2 years of age do not present any tremors or any spontaneous loss of balance while walking, but they do have a hesitating gait.

To further assess the neurological consequences of frataxin depletion and to determine stages in the disease progression, mutant animals were analyzed by several motor skill and behavioral tests. To avoid any influence of memory or adaptation to the test, naive mutant animals from both lines were tested on the accelerating rotarod at each time point: 10, 20 (Fig. $2 a-d$ ), and 30 weeks (data not shown). Although no overt clinical phenotype was observed at 10 weeks, their performance on the rod after $4 \mathrm{~d}$ of trial (genotype factor of the ANOVA test) was significantly poorer than that of matched control littermates [ $44 \%$ (Cb mutants) and $35 \%$ (Br mutants) lower; $p<0.05$ ] (Fig. 2a,c). The defect in performance was confirmed at 20 weeks $(p<0.001$ for $\mathrm{Cb}$ mutants and $p<0.05$ for Br mutants) (Fig. $2 b, d)$. The progression, revealing the ability to improve performance on the motor task during the $4 \mathrm{~d}$ of trial (interaction of the day by genotype factor of the ANOVA test), is significantly reduced for mutants from both lines at 10 weeks ( $35 \%$ reduction for $\mathrm{Br}$ mutants, $p<0.05$; and $51 \%$ reduction for the $\mathrm{Cb}$ line, $p<0.001)$ and worsens at 20 weeks [57\% $(p<0.01)$ and 69\% $(p<0.05)$ reduction, respectively] (Fig. $2 a-d$ ). To follow the phenotype evolution, four mutant animals were subjected to the test at both 20 and 35 weeks. A striking evolution occurs between 20 and 35 weeks for $\mathrm{Cb}$ mutants (Fig. 2e), with complete impairment and inability to improve performance at 35 weeks. In contrast, the performance of Br mutants between 30 and 40 weeks is not significantly different (data not shown). We excluded any influence of muscular weakness on the performance on the rotarod by using a dynamometer at different time points between 10 and 60 weeks. At 60 weeks, $\mathrm{Cb}$ and $\mathrm{Br}$ mutants have muscular strength equivalent to controls $(6.45 \pm 0.75 \mathrm{gm}$ vs $6.89 \pm 1.4 \mathrm{gm}, n=6$; and $6.1 \pm 0.9 \mathrm{gm}$ vs $5.9 \pm 1.5 \mathrm{gm}, n=6$, respectively).

To further assess the evolution of the symptoms, gait parameters of mutant and control mice were evaluated by footprint analysis at 10 (data not shown), 20, 30, and 40 weeks (Fig. $2 f-i$ ). The footprint patterns were assessed quantitatively by five parameters: step length and width, alternation coefficient, linearity, and variation of step length (Fig. $2 f$ ) (see Materials and Methods). At 10 weeks, no parameter was affected. The linearity score, an assessment of ataxia that describes the regularity of direction, was affected at 20 weeks for the Cb mutants ( $p=0.047)$ (Fig. $2 g$ ). Likewise, the alternation coefficient, also revealing a nonuniform gait, is affected for the $\mathrm{Cb}$ mutants at 20 weeks $(p<$ 
0.003 ) (Fig. $2 h$ ). The only parameter significantly increased for both lines at 20 weeks is sigma, describing the regularity in the step length ( $\mathrm{Cb}$ mutants, $p<0.05 ; \mathrm{Br}$ mutants, $p<0.02)$. The three parameters increased progressively at 30 and 40 weeks, indicating a deterioration of the gait. The decrease of the $\sigma$ parameter for the $\mathrm{Cb}$ mutants between 30 and 40 weeks (Fig. 2i) is attributable to the exclusion of the most affected mice, which were no longer able to perform the test. Furthermore, at 40 weeks, mice from both lines weaved from side to side while moving through the tunnel (Fig. 2f) (Cb mutants), using either a wider base ( $\mathrm{Cb}$ mutants only, with $3.2 \pm$ 0.1 vs $2.8 \pm 0.1 \mathrm{~cm} ; p<0.008)$ or shorter steps (Br mutants only, with $4.5 \pm 0.2$ vs $8.5 \pm 0.2 \mathrm{~cm} ; p<0.002)$.

\section{The neurological phenotype is associated with a specific progressive sensory defect}

The function of the peripheral nervous system was assessed by electromyographic exploration after somatosensory stimulation of the sciatic nerve. The $\mathrm{Cb}$ mutants showed a clear defect in the spinal somatosensory response ( $\mathrm{H}$-wave amplitude) at 17 weeks $(0.155 \pm 0.01 \mathrm{mV} ; p<0.002)$, whereas the $\mathrm{Br}$ mutants showed a defect at 32 weeks only $(0.32 \pm 0.006 \mathrm{mV} ; p<$ 0.001 ; lower limit of normal is $0.6 \mathrm{mV}$ ) (Fig. $2 j, k$ ). The latency of the $\mathrm{H}$ wave is normal (data not shown). The $\mathrm{H}$ wave disappears between 28 and 38 weeks for the $\mathrm{Cb}$ mutants, and between 32 and 40 weeks for the Br mutants. The motor evoked potentials and the caudal sensory response (caused almost exclusively by smalldiameter sensory axons) do not differ between controls and mutants for both lines (data not shown). These results indicate that the large myelinated proprioceptive sensory neurons or their afferents are functionally defective, a distinctive pathological feature of FRDA.

\section{Central and peripheral pathologies cause the neurological phenotype} In agreement with the severity of the respective neurological phenotypes, histological analyses reveal more marked anomalies in the $\mathrm{Cb}$ line than in the $\mathrm{Br}$ line. $\mathrm{Cb}$ mutants presented a reduction in the number of cerebellar granule cells beginning between 20 and 30 weeks (Fig. $3 a, b$ ), with the most dramatic worsening between 30 and 40 weeks (Fig. $3 c$ ) but further visible deterioration observed until 1.5 years (data not shown). Despite the loss of granule cells, the overall cerebellar architecture was well preserved, with normal
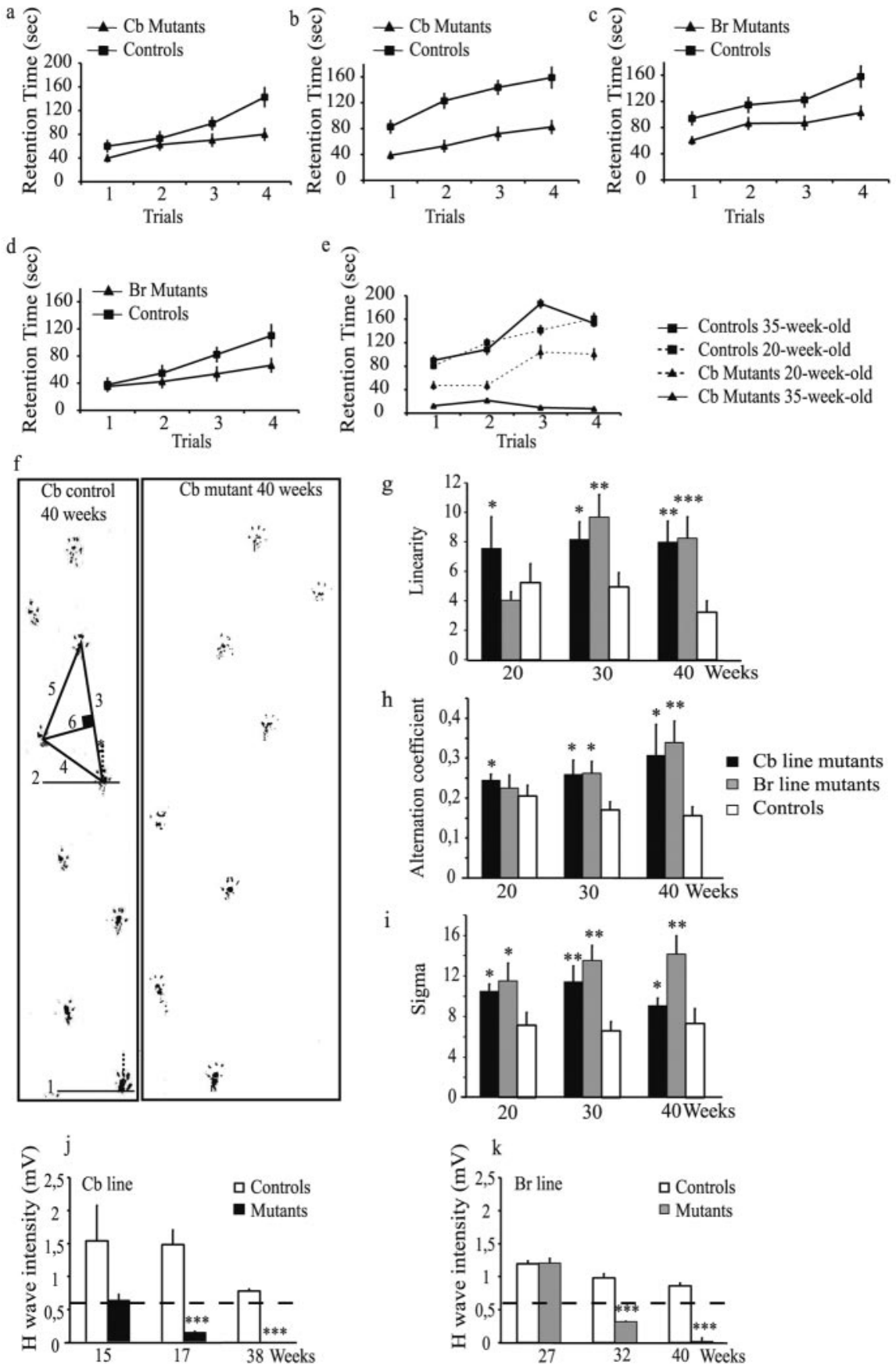

Figure 2. The $\mathrm{Cb}$ and $\mathrm{Br}$ mutants present a progressive neurological phenotype. $a-e$, Rotarod analysis of $\mathrm{Cb}(a, b)$ and $\mathrm{Br}(c, d)$ animals versus control littermates at 10 and 20 weeks, respectively. Different pools of control and mutant mice were used at 10 and 20 weeks ( $n=8$ for each). Impaired performance is observed for both lines at 10 weeks $(p<0.05$ for both lines) and at 20 weeks ( $p<0.05$ for $\mathrm{Br}$ line and $p<0.001$ for $\mathrm{Cb}$ line). e, Comparison of performance of $\mathrm{Cb}$ mice $(n=4)$ at 20 and 35 weeks showing progression of the deficit for the mutants. $f-i$, Footprint analysis of $\mathrm{Br}$ (gray) and $\mathrm{Cb}$ (black) mutants at 20 ( $n=11$ and 6 , respectively), 30 ( $n=11$ for both lines), and 40 weeks ( $n=11$ and 9, respectively) compared with control littermates (white). Because performances of $\mathrm{Br}$ and $\mathrm{Cb}$ control littermates were similar, the results were pooled $(n=16)$. $f$, Footprint patterns were quantitatively assessed for five parameters (see Materials and Methods) as shown on representative footprint patterns of 40week-old control and $\mathrm{Cb}$ mutant animals. The progression of the three parameters is represented graphically: linearity $(g)$, alternation coefficient $(h)$, sigma $(i)$. Asterisks indicate significant difference between control and mutants (Student's $t$ test). ${ }^{*} p<0.05 ;{ }^{* *} p<0.01 ;{ }^{* * *} p<0.001 . j, k$, Electromyographic measurements of the spinal somatosensory evoked response (H wave) in $\mathrm{Br}$ (gray) and $\mathrm{Cb}$ (black) mutants versus control littermates (white) at 27 weeks $(n=6), 32$ weeks $(n=8)$, and 40 weeks $(n=6)$ for Br mice, and at 15 weeks $(n=4)$, 17 weeks $(n=3)$, and 38 weeks $(n=3)$ for Cb mice. The specific absence of the $\mathrm{H}$ wave, indicating a specific dysfunction in the large proprioceptive sensory neurons, is observed in mutants of both lines. The dotted line corresponds to the lower limit of normal $\mathrm{H}$ wave $(0.6 \mathrm{mV})$. The large error bar at 15 weeks for $\mathrm{Cb}$ controls is attributable to one of four animals that showed a very high $\mathrm{H}$-wave amplitude $(2.6 \mathrm{mV}) .{ }^{* *} p<0,001$ (Student's $t$ test). 


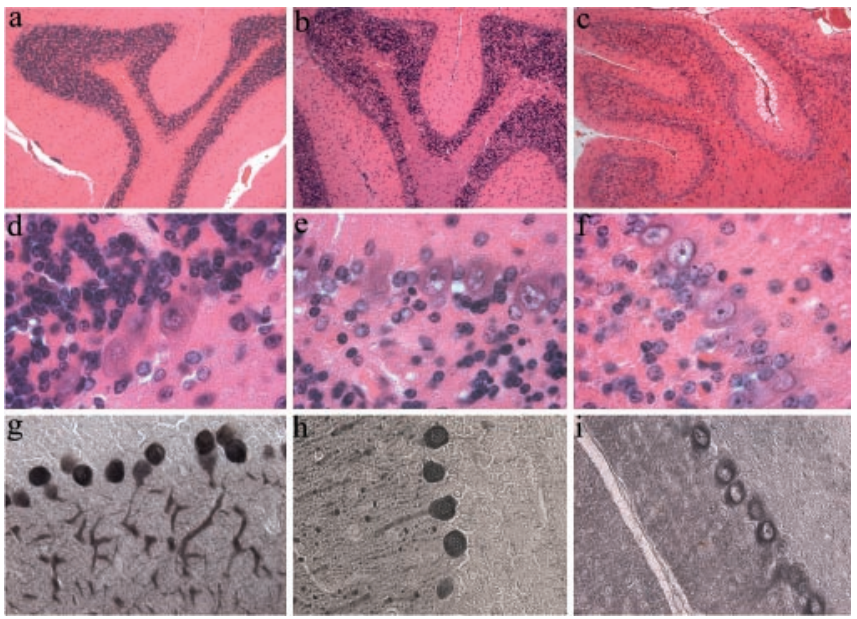

Figure 3. Cerebellar neuropathology in the $\mathrm{Cb}$ mutants shows loss of granule cells and Purkinje cell arborization. $a-f$, H\&E staining of the cerebellum of 20-week-old $(a, d)$ control mice and of 30-week-old $(b, e)$ and 40-week-old $(c, f)$ Cb mutants. Note the reduction of the granule cell layer at 30 weeks $(b)$, which becomes dramatic at 40 weeks $(c)$, and the reduction of Purkinje cell cytoplasm and clarification of the nucleus at 30 and 40 weeks (b-f). $g-i$, Calbindin staining of the cerebellum of a control mouse at 20 weeks $(g)$ and $C b$ mutants at $30(h)$ and 40 (i) weeks. The affected mutants at 30 weeks have reduced dendritic arborizations at 30 weeks $(h)$ and complete loss of arborization at 40 weeks ( $i)$. Original magnification: $a-c, 10 \times$; $d-i, 100 \times$.

foliation and laminar structure. No other neuronal loss was observed. Although no significant Purkinje cell loss or disorganization was noticed (no obvious heterotopia), Purkinje cells present a size reduction of the perikaryon, a clarification of the nucleus (Fig. $3 d-f$ ), and reduced dendritic beginning arborization at 20 weeks, as revealed by anti-calbindin staining (Fig. $3 g-i$ ). The intensity of calbindin immunostaining in Purkinje cells diminished as the mice aged and was very faint by 40 weeks, with almost a complete absence of arborization and very pale cytoplasmic staining caused by the strong reduction of Purkinje cell perikarya. No vacuole was seen in the cerebellum of $\mathrm{Cb}$ mutants at any age.

In contrast, $\mathrm{Br}$ mutants revealed no significant neuronal loss in any areas of the brain observed. Particularly, we did not observe any evidence of spongiform degeneration, as seen previously in the cortex of neuronal-cardiac mice (Puccio et al., 2001), despite significant deletion in the brain (forebrain and midbrain) (Fig. 1b), nor did we observe any loss of granule cells in the cerebellum. The only histological change observed in the brain structure of the Br mutants was a slight reduction of Purkinje cell arborization at $>1$ year of age (data not shown).

The main pathological feature seen in the spinal cord of both mutants was chromatolysis, beginning at 20 weeks in the sensory neurons of the posterior horn. Chromatolysis consists of a loss of basophilic staining of the cell body with peripheral margination of the Nissl substance and is often observed as a consequence of axonal damage. In addition, at 40 weeks, degeneration was also observed for both lines in the spinal motor neuron bodies of the anterior horns and in the nuclei of the Clarke's columns (Fig. $4 a-e)$. At 30 weeks, the posterior columns of $\mathrm{Cb}$ mutants showed a clear hallow demyelination, consistent with axonal damage (Fig. $4 f$ ).

The sensory cell bodies of the DRG reveal a different pattern of degeneration, with the presence of vacuoles at 10 weeks for the $\mathrm{Cb}$ mutants (Fig. $5 a$ ). Fifty percent of the ganglia presented vacuoles in one to eight cells, with no preferential location of the affected ganglia. This degeneration in ganglion cells, confirmed on semi-
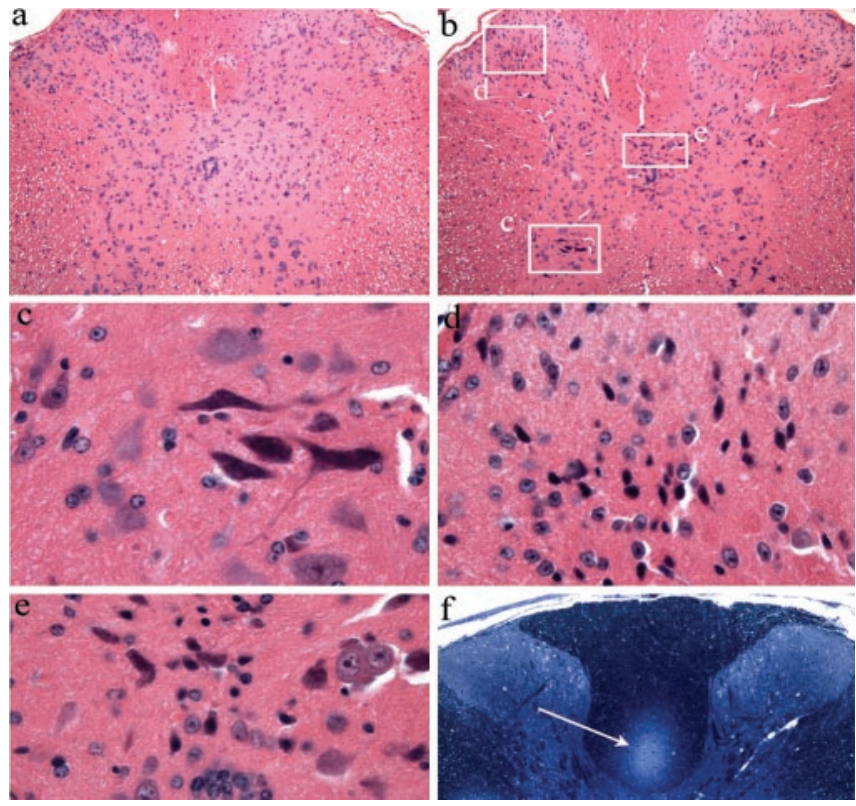

Figure 4. Neuronal degeneration in the spinal cord of the Br mutants and demyelination of the posterior column in the spinal cord of the Cb mutants. $a-e$, Spinal cord section of control $(a)$ and $\mathrm{Br}$ mutant animals at 40 weeks $(b)$ stained with H\&E. Different areas that show degenerated neurons with chromatolysis are magnified: motor neurons of the ventral horn $(c)$ and neurons in the dorsalis nuclei $(d)$ and the Clarke's column $(e)$. Similar neuronal degeneration was found in Cb mutants (data not shown). $f$, Semithin section of a Cb mutant at 30 weeks stained with toluidine blue showing demyelination of posterior column (arrow). Original magnification: $a, b, f, 10 \times ; c-e, 40 \times$.
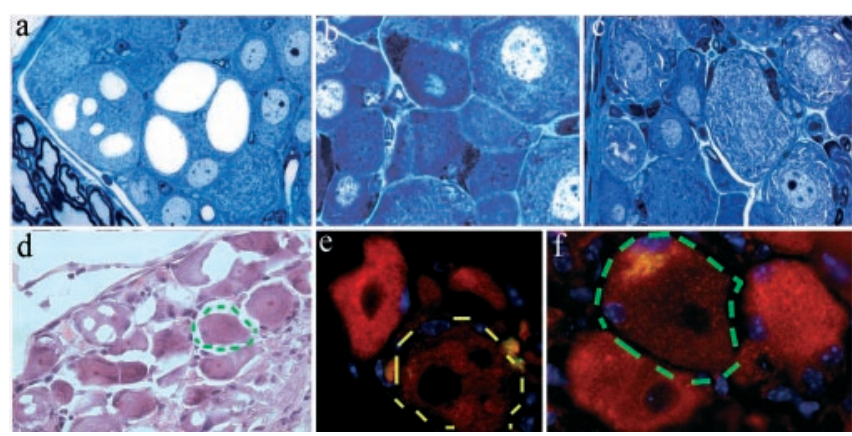

Figure 5. Frataxin-deficient cells in the dorsal root ganglia show degeneration with the presence of vacuoles and lipofuscin accumulation. $a-c$, Semithin section stained with toluidine blue of DRG of a $\mathrm{Cb}$ mutant at 30 weeks, revealing the presence in two large sensory neurons of vacuoles $(a)$, accumulation of peripheral granular deposits corresponding to lipofuscin $(b)$, and swollen Nissl body ( $c$ ). d, H\&E staining of a DRG section of a Cb mutant at 40 weeks, with the following section stained with anti-frataxin antibody (red) and Hoescht (blue) $(e, f)$. Yellow doted line indicates a vacuolated ganglion cell negatively stained for frataxin, and the green dotted line shows a ganglion cell negatively stained for frataxin with accumulation of autofluorescent lipofuscin material (green) $(e, f)$. Original magnification: $d, 40 \times$; all others, $100 \times$.

thin sections stained with toluidine blue at 10, 20, 30, and 40 weeks, appeared to be specific to the large sensory cells, distinguished from small sensory cells by their large and light cytoplasm (Fig. 5a). Noticeably, these are the cells affected in the human disease. In addition, an increased level of intracellular dense deposits reminiscent of lipofuscin was observed at the periphery of the cell (Fig. $5 b$ ) already at 20 weeks and prominently at 40 weeks. Finally, endoplasmic reticulum stress occurred, as revealed by swollen Nissl bodies in some large sensory neurons at 30 weeks (Fig. 5c). Immunohistochemistry demonstrated that the ganglion cells presenting vacuolization and autofluorescent 
lipofuscin accumulation were indeed deleted for frataxin (Fig. $5 d-f$ ). This suggests that in contrast to cerebellar granule cells, ganglion cells can survive longer without frataxin.

Br mutants showed no sign of vacuolization in DRG at 40 weeks or later (2 years old); however, disorganization of the cytoplasm with material pushed to the periphery in the DRG neurons was seen at 20 weeks (data not shown) and presumably corresponds to chromatolysis.

We found no evidence of apoptosis by TUNEL analysis in cerebellum, brain, spinal cord, and DRG of both $\mathrm{Cb}$ and $\mathrm{Br}$ mutants from 20 weeks to $>1$ year.

\section{The pathological mechanism in the DRG is an autophagic process}

Electron microscopy revealed two main types of defects in the DRG cells of the Cb mutants at 10, 20, 30, and 40 weeks: autophagy (giant vacuoles) already present at 10 weeks, and lipofuscin accumulation at 20 weeks, thus corroborating the pathological changes seen by light microscopy. A large number of vesicular structures characteristic of autophagosomes and autolysosomes were observed (Fig. $6 a-g$ ). These vacuoles, bounded by one or more membranes, contain a cytosolic substance and fragments of cellular organelles. In the early stages, macroautophagy resulted in the isolation and engulfment of large areas of cytoplasm (Fig. $6 a$ ). The nascent autophagosomes then fuse with lysosomes, leading to the clarification of the vacuole and the formation of an autolysosome with a single membrane, which continues engulfing cytosol, organelles, and lysosomes (Fig. 6b). Abnormal mitochondria with disorganized cristae and occasional swelling were seen in the vicinity of the autophagic vacuoles (Fig. $6 b, c, e, g$ ). Other mitochondria, usually at a distance from the vacuoles, had a normal appearance. Mitochondrial iron accumulation was never observed. The cytoplasmic sequestration seemed to occur through rough endoplasmic reticulum (RER) membranes, with nascent vacuoles formed by a swollen RER (Fig. 6c) corresponding to the perturbed Nissl body seen on semifine sections (Fig. $5 c$ ). The remaining RER was pushed to the periphery of the cell and had lost its ribosomes (Fig. $6 d$ ). The Golgi apparatus was also disturbed, with dilation of channels and cisterns (Fig. $6 d, e$ ), suggesting that some vacuoles could also originate from Golgi apparatus. Furthermore, the number of lysosomes increased significantly, suggesting a high activation of the degradation processes (Fig. 6d). Undigested material accumulated inside the vacuole, leading to progressive densification (Fig. $6 e-g$ ). At 40 weeks, this dense material completely filled the vacuoles and appeared mineralized (Fig. 6g). Furthermore, lipofuscin accumulation was confirmed (Fig. 6h) and may have resulted from residual undigested electron-dense lipid residues when autolysosomes reached a final point of degradation. Finally, at 30 and 40 weeks, largediameter axons of DRG showed signs of degeneration, whereas the myelin sheath was well preserved (Fig. 6i).

\section{Neuronal tissues have moderate iron-sulfur deficiency and iron deposit}

To assess whether the neuropathology observed was associated with a specific deficit of ISC-containing enzymes and iron accumulation as described for the cardiac tissues of both FRDA patients and animal models, biochemical analysis of the ISCcontaining enzyme SDH compared with COX activities as well as histological iron staining were performed. Analyses of spinal cord, cerebellum, brain stem, and brain were performed for both lines $(n=4)$ at different ages, ranging from 10 weeks to $>1$ year for iron detection and at 40 weeks for enzymatic measurements.

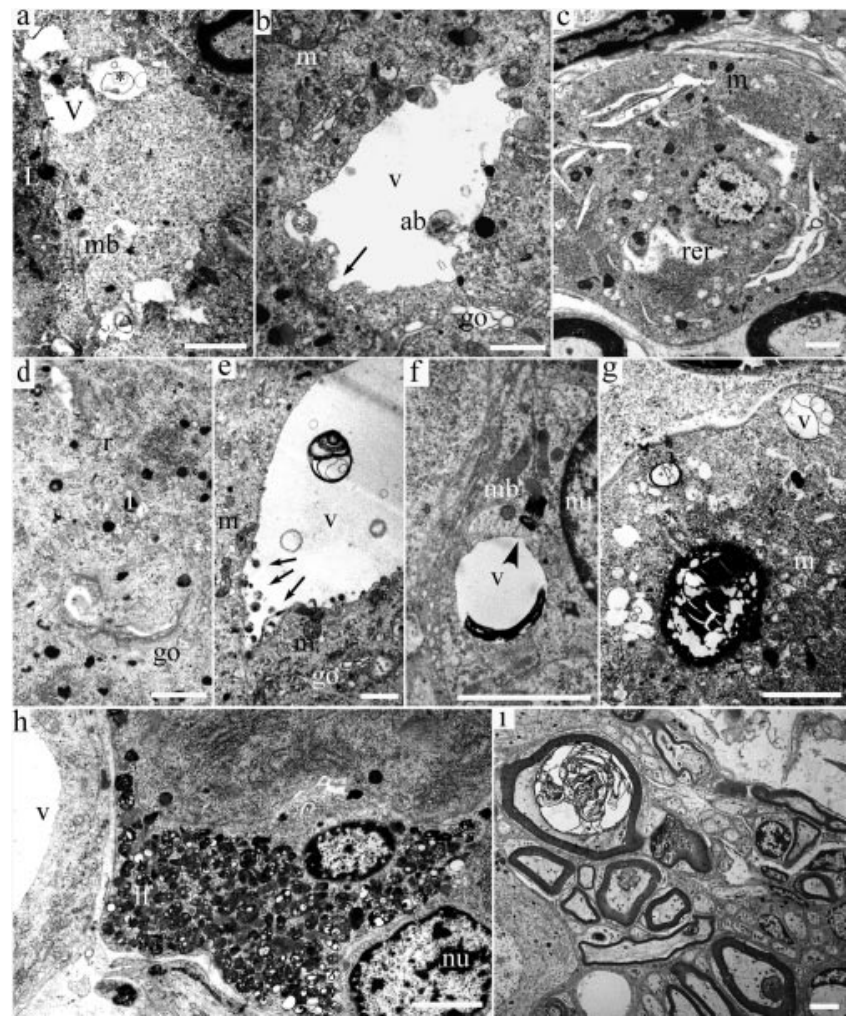

Figure 6. Autophagic features and lipofuscin accumulation in the DRG of $C b$ mutants; electron microscopy on Cb mutant DRG at $30(e, f, i)$ and $40(a-d, g, h)$ weeks. $a$, Electron-lucent cytoplasmic area with fibrillary material, delimited by a membrane-like structure. Focal cytoplasmic breakdowns occur, and several autophagic vacuoles develop at the periphery sequestering portions of cytoplasm that contain lipopigments, glycogen, and concentric bodies with membrane remnants (asterisk). Lysosomes are present at the periphery. $b, \mathrm{~A}$ large vacuole of autophagy shows membrane activity and pinocytosis (arrow) and contains projections of cytoplasmic matrix and ribosomes (white asterisks) that are either attached to the inner membrane or freely floating. Autophagic bodies, a swollen Golgi, and affected mitochondria are seen. c, A marked dilatation of the RER generates vacuoles containing remnants of ergastoplasm and lysosomes. $d-g$, Various profiles of autophagy. $d$, The Golgi cisterns are dilated or compacted, and the RER is pushed to the periphery of the cell with polyribosomes lying free in the cytoplasm. Lysosomes are significantly increased in size. $e$, High membrane activity of a vacuole with numerous pinocytotic vesicles coating the inner surface (arrows) and electron-dense residual myelinoid material loaded. Some mitochondria are degenerating (adjacent to the vacuole), whereas others have a normal appearance (bottom). Profiles as seen in $a-e$ were detected in Cb mutants from 10 to 40 weeks. $f$, At 30 weeks, dense material accumulation is seen. Note the budding (black arrowhead), membrane stacking, and autophagosomes adjacent to the vacuole, suggesting high autophagy activity. $g$, Progressive filling of the vacuole with dense deposits having a mineralized appearance. Autolysosomes containing membrane remnants are seen. $h$, Peripheral massive lipofuscin accumulation, made of numerous dense granules with various shapes. $i$, The large-diameter axons are degenerating in the absence of myelin alteration. Scale bars, $2.5 \mu \mathrm{m}$. ab, Autophagic body; go, Golgi; L, lysosome; If, lipofuscin; mb, membrane; m, mitochondria; nu, nucleus; r, polyribosome; rer, rough endoplasmic reticulum; v, vacuole.

Previous studies on heart biopsies from FRDA patients and mice models have demonstrated that COX activity is not altered as a consequence of frataxin deficiency and can be used as an internal control for SDH (complex II) activity (Rotig et al., 1997; Puccio et al., 2001). The most significant difference in SDH activity was found in the spinal cord of $\mathrm{Br}$ line mutants with a $40 \%$ reduction of SDH/COX activity ratios compared with control littermates. A suggestive reduction of SDH/COX activity ratios was also seen in the cerebellum of $\mathrm{Cb}$ mutants and may be partially masked by the massive cerebellar neuron loss seen at this age (data not shown). Very few iron deposits were seen in either mutant, even late in the 
disease, with rare deposits seen in the dorsal horn, brain stem, cerebellar granular layer, and cortex (data not shown), confirming that this is a late event in the pathological process $(\mathrm{H}$. Seznec, D. Simon, L. Monassier, P. Criqui-Filipe, A. Gansmuller, P. Rustin, M. Koenig, and H. Puccio, unpublished observations), and is reminiscent of the situation in FRDA patients (Lamarche et al., 1993; Waldvogel et al., 1999; Bradley et al., 2000).

\section{Discussion}

Friedreich ataxia is a slowly progressive neurodegenerative disorder caused by severely reduced levels of frataxin. Several attempts have been made to produce an authentic model for the neurological symptoms of FRDA, but until now, no mouse model parallels the progressive neurological human disease (Puccio et al., 2001; Miranda et al., 2002). Through spatially and temporally controlled conditional FRDA gene inactivation, we have generated two different mouse models that developed the most prominent features of the human disease: a slowly progressive mixed cerebellar and sensory ataxia associated with a progressive loss of proprioception and absence of motor involvement. Interestingly, the neurological deficit is more severe in the $\mathrm{Cb}$ line than in the $\mathrm{Br}$ line, leading to loss of spontaneous ambulation by 1 year of age, despite the more restricted frataxin deficiency seen by Western blot analysis, indicating that the difference most likely arises from a selective neuronal sensitivity to frataxin deletion.

These mouse models also parallel the human disease at the histopathological level. The models have degeneration of the posterior columns of the spinal cord that appear translucent, because of demyelination and loss of fibers, and severe lesions of neurons in Clarke's columns, hallmarks of FRDA disease. In addition, the $\mathrm{Cb}$ mutants have specific damage to the large sensory neuron cell bodies in the DRG, another distinctive feature of FRDA. The time of occurrence of these lesions suggests that as in patients, the anomalies observed in the neuronal cell bodies of the DRG are a primary event, whereas the neuronal loss in Clarke's column and the degeneration in posterior column might be secondary events.

Our mouse models also show some features that are a departure from FRDA, in particular the extent of cerebellar pathology. Cerebellar atrophy is inconsistently observed in FRDA; some patients have mild loss of Purkinje cells with occasional axonal torpedoes late in the disease course (Koeppen, 1991). Only the $\mathrm{Cb}$ mutant shows an early loss of dendritic Purkinje cell arborization and abnormal cell morphology with reduction of the perikaryon and clarification of the nucleus, in agreement with the high expression of the Prp 28.6 transgene in this cell type, compared with the Prp 28.4 transgene (Weber et al., 2001). In addition, the $\mathrm{Cb}$ line has a progressive cerebellar granule cell loss, not characteristic of FRDA. Although the granule cell loss is possibly a direct consequence of complete absence of frataxin in these cells, it is equally possible that they are secondary to Purkinje cell anomalies or to cerebellar afferent alterations.

The progressive neurodegeneration of the DRG in the $\mathrm{Cb}$ line certainly represents an excellent model for unraveling the pathological cascade leading to neuronal death in FRDA. Several cell death pathways can be activated during neurodegeneration, including apoptosis and the more recently accepted mechanism involving autophagy (Xue et al., 1999; Doughty et al., 2000; Yue et al., 2002). Although oxidative insult to cultured cells from FRDA patients results mostly in apoptosis (Wong et al., 1999; Santos et al., 2001), no evidence for such a cell death mechanism in vivo has been provided so far. In particular, the apoptosis process could not be detected in either the complete frataxin knock-out (Cossee et al., 2000) or the conditional mouse model (Puccio et al., 2001).
Surprisingly, we have observed that the degenerative mechanism involved in the dorsal root ganglia neurons is an autophagic process, leading to removal and degradation of damaged cytosolic proteins and organelles. Autophagy is characterized by the presence of autophagic vacuoles and autophagosomes that are formed by rearrangement of subcellular membranes (rough endoplasmic reticulum or trans-Golgi system) to sequester cytosolic constituents and organelles and traffic them to lysosomes for degradation (Reggiori and Klionsky, 2002). Different steps of the autophagic process were clearly seen in the large myelinated DRG neurons of the $\mathrm{Cb}$ mutants. In addition to autophagosomes, lipofuscin accumulation was also observed in these neurons. Lipofuscin is composed of proteins, lipids, carbohydrates, and metals, particularly iron (Brunk and Terman, 2002) derived from mitochondria and metalloproteins having incomplete autophagocytosis and lysosomal degradation, thereby leading to their accumulation. Because lipofuscin accumulation has been reported both in the DRG and in the cardiomyocytes of FRDA patients (Lamarche et al., 1980, 1982; Larnaout et al., 1997), this cellular response is certainly a close consequence of frataxin deficiency. Moreover, both processes appear to be cell autonomous and progressive, because the ganglion cell neurons that show autophagosomes and lipofuscin accumulation are deleted for frataxin. Furthermore, the concurrent activation of both processes could explain the lack of detectable iron deposits within the DRG.

The formation of autophagic vacuoles is possibly stimulated by various intracellular and extracellular stress situations, including amino acid starvation, aggregation of damaged proteins, and accumulation of damaged organelles (Petiot et al., 2002). In keeping with this, very recent microarray data suggest a specific sulfur amino acid (SAA) metabolism defect in FRDA cell models (Tan et al., 2003). This specific defect resulting in SAA starvation may be directly linked to the primary role of frataxin in the early steps of ISC biosynthesis and might trigger the autophagic process seen in the DRG. Furthermore, oxidative stress is highly suspected to play an important role in the pathogenesis of FRDA, with elevated levels of oxidative stress markers in patients, including urine 8-hydroxy-2'-deoxyguanosine and serum malondialdehyde (indicative of DNA damage and lipid peroxidation, respectively). Lipofuscin, which is a product of lipid peroxidation, is believed to be responsible, to a large extent, for the cytotoxic effects observed during oxidative stress (Yin et al., 1995; Nilsson et al., 1997), and its formation might represent the ultimate step in FRDA neurodegeneration. A mouse model for ataxia with vitamin E deficiency (AVED) (Yokota et al., 2001), a disease neurologically very similar to FRDA, also presents lipofuscin accumulation within the DRG, indicative of massive membrane oxidative stress resulting from the absence of vitamin $\mathrm{E}$. The AVED mice do not present autophagic neuronal degeneration, however, presumably because of the very late onset of the phenotype, after 1 year of age.

Finally, both the present neurological mouse models and the previous FRDA mouse models that we have generated (Puccio et al., 2001) clearly show accumulation of damaged mitochondria as a direct consequence of frataxin deficiency. Interestingly, the cardiomyocytes in the cardiac model appear to compensate for lack of frataxin by mitochondrial proliferation and cellular hypertrophy without entering an autophagic process. In contrast, DRG neurons appear to survive without frataxin for a longer period by intracellular removal of damaged organelles and proteins through the autophagic and lysosomal pathways. Therefore, the slowly progressive phenotype of the inducible neurological models would reflect the progressive nature of the autophagic 
process, proposed as a protective mechanism for the elimination of defective mitochondria with dysfunctional inner membranes (Lemasters et al., 1998).

In conclusion, these models represent excellent tools for unraveling the pathological cascade of FRDA and testing compounds that interfere with the degenerative process, such as anti-oxidants, which are good pharmacological candidates. Furthermore, these models should prove useful for the investigation of neurodegenerative mechanisms characterized by delayed onset and slow progression over years or decades. Indeed, oxidative stress and mitochondrial dysfunction, as well as activation of autophagy in several of the major human neurodegenerative disorders, including Alzheimer's disease (Nixon et al., 2000; Hirai et al., 2001; Perry et al., 2002), Parkinson's disease (Anglade et al., 1997; Meredith et al., 2002), and Huntington's disease (Kegel et al., 2000), have been reported repeatedly, although their role in these pathologies is still discussed. The novel spatiotemporally controlled conditional gene-targeting approach that we have used is particularly adapted to study the mechanisms of lateonset and slowly progressive neurodegeneration and is amenable to large experimental flexibility through modulation at will of the timing of induction.

\section{References}

Anglade P, Vyas S, Javoy-Agid F, Herrero MT, Michel PP, Marquez J, MouattPrigent A, Ruberg M, Hirsch EC, Agid Y (1997) Apoptosis and autophagy in nigral neurons of patients with Parkinson's disease. Histol Histopathol 12:25-31.

Bradley JL, Blake JC, Chamberlain S, Thomas PK, Cooper JM, Schapira AH (2000) Clinical, biochemical and molecular genetic correlations in Friedreich's ataxia. Hum Mol Genet 9:275-282.

Brunk UT, Terman A (2002) Lipofuscin: mechanisms of age-related accumulation and influence on cell function. Free Radic Biol Med 33:611-619.

Campuzano V, Montermini L, Molto MD, Pianese L, Cossee M, Cavalcanti F, Monros E, Rodius F, Duclos F, Monticelli A, et al (1996) Friedreich's ataxia: autosomal recessive disease caused by an intronic GAA triplet repeat expansion. Science 271:1423-1427.

Chantrel-Groussard K, Geromel V, Puccio H, Koenig M, Munnich A, Rotig A, Rustin P (2001) Disabled early recruitment of antioxidant defenses in Friedreich's ataxia. Hum Mol Genet 10:2061-2067.

Clark HB, Burright EN, Yunis WS, Larson S, Wilcox C, Hartman B, Matilla A, Zoghbi HY, Orr HT (1997) Purkinje cell expression of a mutant allele of SCA1 in transgenic mice leads to disparate effects on motor behaviors, followed by a progressive cerebellar dysfunction and histological alterations. J Neurosci 17:7385-7395.

Cossee M, Puccio H, Gansmuller A, Koutnikova H, Dierich A, LeMeur M, Fischbeck K, Dolle P, Koenig M (2000) Inactivation of the Friedreich ataxia mouse gene leads to early embryonic lethality without iron accumulation. Hum Mol Genet 9:1219-1226.

Doughty ML, De Jager PL, Korsmeyer SJ, Heintz N (2000) Neurodegeneration in lurcher mice occurs via multiple cell death pathways. J Neurosci 20:3687-3694.

Duby G, Foury F, Ramazzotti A, Herrmann J, Lutz T (2002) A non-essential function for yeast frataxin in iron-sulfur cluster assembly. Hum Mol Genet 11:2635-2643.

Emond M, Lepage G, Vanasse M, Pandolfo M (2000) Increased levels of plasma malondialdehyde in Friedreich ataxia. Neurology 55:1752-1753.

Finocchiaro G, Baio G, Micossi P, Pozza G, di Donato S (1988) Glucose metabolism alterations in Friedreich's ataxia. Neurology 38:1292-1296.

Gabe M (1968) Techniques histologiques. Paris: Ed Masson et Cie.

Harding AE (1981) Friedreich's ataxia: a clinical and genetic study of 90 families with an analysis of early diagnostic criteria and intrafamilial clustering of clinical features. Brain 104:589-620.

Harding AE, Hewer RL (1983) The heart disease of Friedreich's ataxia: a clinical and electrocardiographic study of 115 patients, with an analysis of serial electrocardiographic changes in 30 cases. Q J Med 52:489-502.

Hirai K, Aliev G, Nunomura A, Fujioka H, Russell RL, Atwood CS, Johnson AB, Kress Y, Vinters HV, Tabaton M, Shimohama S, Cash AD, Siedlak SL,
Harris PL, Jones PK, Petersen RB, Perry G, Smith MA (2001) Mitochondrial abnormalities in Alzheimer's disease. J Neurosci 21:3017-3023.

Hughes JT, Brownell B, Hewer RL (1968) The peripheral sensory pathway in Friedreich's ataxia. An examination by light and electron microscopy of the posterior nerve roots, posterior root ganglia, and peripheral sensory nerves in cases of Friedreich's ataxia. Brain 91:803-818.

Huynen MA, Snel B, Bork P, Gibson TJ (2001) The phylogenetic distribution of frataxin indicates a role in iron-sulfur cluster protein assembly. Hum Mol Genet 10:2463-2468.

Kegel KB, Kim M, Sapp E, McIntyre C, Castano JG, Aronin N, DiFiglia M (2000) Huntingtin expression stimulates endosomal-lysosomal activity, endosome tubulation, and autophagy. J Neurosci 20:7268-7278.

Koeppen AH (1991) The Purkinje cell and its afferents in human hereditary ataxia. J Neuropathol Exp Neurol 50:505-514.

Koeppen AH (1998) The hereditary ataxias. J Neuropathol Exp Neurol 57:531-543.

Koutnikova H, Campuzano V, Foury F, Dolle P, Cazzalini O, Koenig M (1997) Studies of human, mouse and yeast homologues indicate a mitochondrial function for frataxin. Nat Genet 16:345-351.

Lamarche J, Luneau C, Lemieux B (1982) Ultrastructural observations on spinal ganglion biopsy in Friedreich's ataxia: a preliminary report. Can J Neurol Sci 9:137-139.

Lamarche JB, Cote M, Lemieux B (1980) The cardiomyopathy of Friedreich's ataxia morphological observations in 3 cases. Can J Neurol Sci 7:389-396.

Lamarche JB, Shapcott D, Cote M, Lemieux B (1993) Cardiac iron deposits in Friedreich's ataxia. In: Handbook of cerebellar diseases. (Lechtenberg R, ed), pp 453-458. New York: Marcel Dekker.

Larnaout A, Belal S, Zouari M, Fki M, Ben Hamida C, Goebel HH, Ben Hamida M, Hentati F (1997) Friedreich's ataxia with isolated vitamin E deficiency: a neuropathological study of a Tunisian patient. Acta Neuropathol (Berl) 93:633-637.

Lemasters JJ, Nieminen AL, Qian T, Trost LC, Elmore SP, Nishimura Y, Crowe RA, Cascio WE, Bradham CA, Brenner DA, Herman B (1998) The mitochondrial permeability transition in cell death: a common mechanism in necrosis, apoptosis and autophagy. Biochim Biophys Acta 1366:177-196.

Lutz T, Westermann B, Neupert W, Herrmann JM (2001) The mitochondrial proteins Ssq1 and Jac1 are required for the assembly of iron sulfur clusters in mitochondria. J Mol Biol 307:815-825.

Meredith GE, Totterdell S, Petroske E, Santa Cruz K, Callison Jr RC, Lau YS (2002) Lysosomal malfunction accompanies alpha-synuclein aggregation in a progressive mouse model of Parkinson's disease. Brain Res 956:156-165.

Metzger D, Chambon P (2001) Site- and time-specific gene targeting in the mouse. Methods 24:71-80.

Miranda CJ, Santos MM, Ohshima K, Smith J, Li L, Bunting M, Cossee M, Koenig M, Sequeiros J, Kaplan J, Pandolfo M (2002) Frataxin knockin mouse. FEBS Lett 512:291-297.

Muhlenhoff U, Richhardt N, Ristow M, Kispal G, Lill R (2002) The yeast frataxin homolog Yfhlp plays a specific role in the maturation of cellular $\mathrm{Fe} / \mathrm{S}$ proteins. Hum Mol Genet 11:2025-2036.

Nilsson E, Ghassemifar R, Brunk UT (1997) Lysosomal heterogeneity between and within cells with respect to resistance against oxidative stress. Histochem J 29:857-865.

Nixon RA, Cataldo AM, Mathews PM (2000) The endosomal-lysosomal system of neurons in Alzheimer's disease pathogenesis: a review. Neurochem Res 25:1161-1172.

Oppenheimer D, Esiri M (1992) Disease of the basal ganglia, cerebellum and motor neurons. In: Greenfield's neuropatholog, Ed 5 (Adams JH, Corsellis J, Duchen LW, eds), pp 988-1045. London: Arnold.

Perry G, Taddeo MA, Nunomura A, Zhu X, Zenteno-Savin T, Drew KL, Shimohama S, Avila J, Castellani RJ, Smith MA (2002) Comparative biology and pathology of oxidative stress in Alzheimer and other neurodegenerative diseases: beyond damage and response. Comp Biochem Physiol C Toxicol Pharmacol 133:507-513.

Petiot A, Pattingre S, Arico S, Meley D, Codogno P (2002) Diversity of signaling controls of macroautophagy in mammalian cells. Cell Struct Funct 27:431-441.

Puccio H, Koenig M (2002) Friedreich ataxia: a paradigm for mitochondrial diseases. Curr Opin Genet Dev 12:272-277.

Puccio H, Simon D, Cossee M, Criqui-Filipe P, Tiziano F, Melki J, Hindelang 
C, Matyas R, Rustin P, Koenig M (2001) Mouse models for Friedreich ataxia exhibit cardiomyopathy, sensory nerve defect and Fe-S enzyme deficiency followed by intramitochondrial iron deposits. Nat Genet 27:181-186.

Reggiori F, Klionsky DJ (2002) Autophagy in the eukaryotic cell. Eukaryot Cell 1:11-21.

Rotig A, de Lonlay P, Chretien D, Foury F, Koenig M, Sidi D, Munnich A, Rustin P (1997) Aconitase and mitochondrial iron-sulphur protein deficiency in Friedreich ataxia. Nat Genet 17:215-217.

Rustin P, Chretien D, Bourgeron T, Gerard B, Rotig A, Saudubray JM, Munnich A (1994) Biochemical and molecular investigations in respiratory chain deficiencies. Clin Chim Acta 228:35-51.

Santos MM, Ohshima K, Pandolfo M (2001) Frataxin deficiency enhances apoptosis in cells differentiating into neuroectoderm. Hum Mol Genet 10:1935-1944.

Schulz JB, Dehmer T, Schols L, Mende H, Hardt C, Vorgerd M, Burk K, Matson W, Dichgans J, Beal MF, Bogdanov MB (2000) Oxidative stress in patients with Friedreich ataxia. Neurology 55:1719-1721.

Tan G, Napoli E, Taroni F, Cortopassi G (2003) Decreased expression of genes involved in sulfur amino acid metabolism in frataxin-deficient cells. Hum Mol Genet 12:1699-1711.
Waldvogel D, Gelderen Pv, Hallett M (1999) Increased iron in the dentate nucleus of patients with Friedreich's ataxia. Ann Neurol 46:123-125.

Weber P, Metzger D, Chambon P (2001) Temporally controlled targeted somatic mutagenesis in the mouse brain. Eur J Neurosci 14:1777-1783.

Wong A, Yang J, Cavadini P, Gellera C, Lonnerdal B, Taroni F, Cortopassi G (1999) The Friedreich's ataxia mutation confers cellular sensitivity to oxidant stress which is rescued by chelators of iron and calcium and inhibitors of apoptosis. Hum Mol Genet 8:425-430.

Xue L, Fletcher GC, Tolkovsky AM (1999) Autophagy is activated by apoptotic signaling in sympathetic neurons: an alternative mechanism of death execution. Mol Cell Neurosci 14:180-198.

Yin D, Yuan X, Brunk UT (1995) Test-tube simulated lipofuscinogenesis. Effect of oxidative stress on autophagocytotic degradation. Mech Ageing Dev 81:37-50.

Yokota T, Igarashi K, Uchihara T, Jishage K, Tomita H, Inaba A, Li Y, Arita M, Suzuki H, Mizusawa H, Arai H (2001) Delayed-onset ataxia in mice lacking alpha-tocopherol transfer protein: model for neuronal degeneration caused by chronic oxidative stress. Proc Natl Acad Sci USA 98:15185-15190.

Yue Z, Horton A, Bravin M, DeJager PL, Selimi F, Heintz N (2002) A novel protein complex linking the delta 2 glutamate receptor and autophagy: implications for neurodegeneration in lurcher mice. Neuron 35:921-933. 